\title{
The role of the $\beta$-ionone ring in the photochemical reaction of rhodopsin
}

\author{
Robert Send \\ Institut für Physikalische Chemie, Universität Karlsruhe, \\ Kaiserstrasse 12, 76128 Karlsruhe, Germany \\ Dage Sundholm* \\ Department of Chemistry, P.O. Box 55 (A.I. Virtanens plats 1), \\ FIN-00014 University of Helsinki, Finland
}

*Electronic address: sundholm@chem.helsinki.fi, FAX:+358-9-19150169, Phone: +358-9-19150176 
Table I: The atomic coordinates (in bohr) of the ground state of 11-cis-retinal protonated Schiff base obtained at the B3LYP/TZVP level.

\begin{tabular}{|c|c|c|c|}
\hline Atom & $\mathrm{x}$ & $\mathrm{y}$ & $\mathrm{z}$ \\
\hline $\mathrm{C}$ & 8.18923803319523 & -1.05918217053671 & 1.23437751911854 \\
\hline $\mathrm{C}$ & 10.11610217789743 & 0.90799943400538 & 2.19324548863060 \\
\hline $\mathrm{C}$ & 10.82977086985440 & 2.84844310313971 & 0.19573355108346 \\
\hline $\mathrm{C}$ & 8.47997850810503 & 4.28782819564588 & -0.65042838131928 \\
\hline $\mathrm{C}$ & 6.16791113357047 & 2.67362422646447 & -1.07867681325705 \\
\hline $\mathrm{C}$ & 5.98660033996453 & 0.26527118865009 & -0.17280708133055 \\
\hline $\mathrm{C}$ & 7.18964710944312 & -2.49856978056915 & 3.56775079240747 \\
\hline $\mathrm{C}$ & 9.50489750738378 & -2.95545560987053 & -0.55355429987733 \\
\hline $\mathrm{C}$ & 4.17029660831732 & 3.97983885092179 & -2.62840708694035 \\
\hline $\mathrm{C}$ & 3.80041109859227 & -1.33081022710472 & -0.57299338113256 \\
\hline $\mathrm{C}$ & 1.30168734888815 & -0.68035151295263 & -0.55001639969335 \\
\hline $\mathrm{C}$ & -0.78033541318836 & -2.35528024641402 & -0.91523960351887 \\
\hline $\mathrm{C}$ & -0.38614119440333 & -5.09148964310075 & -1.59211467026366 \\
\hline $\mathrm{C}$ & -3.20273486909523 & -1.36508884022695 & -0.59263279402930 \\
\hline $\mathrm{C}$ & -5.42623652385572 & -2.76888758001188 & -0.87906372730391 \\
\hline $\mathrm{C}$ & -7.96619960979780 & -2.13369003444154 & -0.58441725724666 \\
\hline $\mathrm{C}$ & -9.14522298483632 & 0.12667489451710 & 0.16223476813825 \\
\hline $\mathrm{C}$ & -7.64761236229870 & 2.44262936644037 & 0.83541292503087 \\
\hline $\mathrm{C}$ & -11.80791307143308 & 0.11721312530109 & 0.25672479913683 \\
\hline $\mathrm{C}$ & -13.32901425505549 & 2.11508497013523 & 0.93734088795789 \\
\hline $\mathrm{N}$ & -15.84995479594369 & 2.03287041007791 & 1.00053178253803 \\
\hline $\mathrm{H}$ & 11.78941088406052 & -0.09177682105664 & 2.87825464629435 \\
\hline $\mathrm{H}$ & 9.31072759544856 & 1.89126884339609 & 3.82798610905408 \\
\hline $\mathrm{H}$ & 11.70366330047775 & 1.92254614931103 & -1.43108987828278 \\
\hline $\mathrm{H}$ & 12.23237575507935 & 4.16949803599870 & 0.93570860591413 \\
\hline $\mathrm{H}$ & 6.11961877065258 & -1.24825801483815 & 4.81429610555266 \\
\hline
\end{tabular}


Table II: Continued. The atomic coordinates (in bohr) of the ground state of 11-cis-retinal protonated Schiff base obtained at the B3LYP/TZVP level.

\begin{tabular}{|c|c|c|c|}
\hline Atom & $\mathrm{x}$ & $\mathrm{y}$ & $\mathrm{z}$ \\
\hline $\mathrm{H}$ & 5.97638317610871 & -4.08955597907378 & 3.06327323083289 \\
\hline $\mathrm{H}$ & 8.77506193211496 & -3.26185732697434 & 4.64751851615810 \\
\hline $\mathrm{H}$ & 11.00432669115505 & -3.95390119257816 & 0.45573391094896 \\
\hline $\mathrm{H}$ & 8.19591497898681 & -4.38449812498665 & -1.26589639267555 \\
\hline $\mathrm{H}$ & 10.34304910415424 & -2.01722505554598 & -2.18615709251862 \\
\hline $\mathrm{H}$ & 5.06123169679285 & 5.07556103645302 & -4.13365410881764 \\
\hline $\mathrm{H}$ & 2.81806192565035 & 2.69345250927399 & -3.48683478240414 \\
\hline $\mathrm{H}$ & 3.11938046996588 & 5.33957790525311 & -1.47055841338995 \\
\hline $\mathrm{H}$ & 4.23846867345305 & -3.32216872157432 & -0.76950658457951 \\
\hline $\mathrm{H}$ & 0.80351530886220 & 1.25693094682938 & -0.11486802098867 \\
\hline $\mathrm{H}$ & -1.48695590408185 & -5.60559288704697 & -3.25882655274113 \\
\hline $\mathrm{H}$ & 1.57500370495532 & -5.52599245841283 & -2.01047535430777 \\
\hline $\mathrm{H}$ & -0.98970884630704 & -6.32239439815350 & -0.04714936093190 \\
\hline $\mathrm{H}$ & -3.30568365367910 & 0.60970343721829 & -0.08712900837182 \\
\hline $\mathrm{H}$ & -5.15821724883997 & -4.72328315896474 & -1.43960866956880 \\
\hline $\mathrm{H}$ & -9.25999083680398 & -3.67012788243077 & -0.99556525052107 \\
\hline $\mathrm{H}$ & -8.81462741099510 & 4.01376948046974 & 1.45678883307258 \\
\hline $\mathrm{H}$ & -6.31607588321328 & 2.02016723275930 & 2.35434441263677 \\
\hline $\mathrm{H}$ & -12.74549749607689 & -1.63435078168313 & -0.25898831546612 \\
\hline $\mathrm{H}$ & -12.53543852824860 & 3.92211688736428 & 1.47537147063077 \\
\hline $\mathrm{H}$ & -16.87590615090966 & 3.55051279177557 & 1.51372749225262 \\
\hline $\mathrm{H}$ & -16.81133278300770 & 0.45229284753660 & 0.53856826244082 \\
\hline $\mathrm{H}$ & 8.86773892476435 & 5.37454099124850 & -2.36529709491113 \\
\hline $\mathrm{H}$ & 7.97203774364774 & 5.70561896920645 & 0.78290598471643 \\
\hline $\mathrm{H}$ & -6.55299935950742 & 3.08774800101796 & -0.79214795380074 \\
\hline
\end{tabular}


Table III: The atomic coordinates (in bohr) of the first excited state of 11-cis-retinal protonated Schiff base obtained at the B3LYP/TZVP level using the TDDFT method.

\begin{tabular}{|c|c|c|c|}
\hline Atom & $\mathrm{x}$ & $\mathrm{y}$ & $\mathrm{z}$ \\
\hline $\mathrm{C}$ & 7.14488412097931 & 1.17269362123241 & 2.03771954052521 \\
\hline $\mathrm{C}$ & 9.19742410095301 & 3.13998662885520 & 1.37150460626497 \\
\hline $\mathrm{C}$ & 11.02552922247685 & 2.21682638629618 & -0.65156887171816 \\
\hline $\mathrm{C}$ & 9.56342072442683 & 1.58765133937969 & -3.05342777170636 \\
\hline $\mathrm{C}$ & 7.16344520099744 & 0.18907497637689 & -2.67963891882741 \\
\hline $\mathrm{C}$ & 6.02899456303834 & -0.07466830692759 & -0.25113258188713 \\
\hline $\mathrm{C}$ & 5.08711301649961 & 2.45706021616673 & 3.66408445760788 \\
\hline $\mathrm{C}$ & 8.32670949152112 & -1.02177563247900 & 3.64759898361946 \\
\hline $\mathrm{C}$ & 5.99005673253182 & -0.95069171121966 & -4.95003028511335 \\
\hline $\mathrm{C}$ & 3.82469122212637 & -1.80333712020123 & 0.06045181966390 \\
\hline $\mathrm{C}$ & 1.40145729610243 & -1.12391265232347 & -0.05430460571147 \\
\hline $\mathrm{C}$ & -0.79611402370425 & -2.79563041822150 & 0.25808002507279 \\
\hline $\mathrm{C}$ & -0.27180789651176 & -5.54318113732398 & 0.74487364193340 \\
\hline $\mathrm{C}$ & -3.13728439853271 & -1.74827070356120 & 0.11135877382592 \\
\hline $\mathrm{C}$ & -5.50872980005642 & -3.03986520840258 & 0.37383659466732 \\
\hline $\mathrm{C}$ & -7.95111776763006 & -2.13991487648250 & 0.28823605209786 \\
\hline $\mathrm{C}$ & -9.00221123016713 & 0.30068836972568 & -0.06127455140800 \\
\hline $\mathrm{C}$ & -7.37638607695200 & 2.59954123495294 & -0.44221891651229 \\
\hline $\mathrm{C}$ & -11.65899340434221 & 0.48314816172954 & -0.03261970434634 \\
\hline $\mathrm{C}$ & -13.10179351413085 & 2.62388937560612 & -0.32985484940534 \\
\hline $\mathrm{N}$ & -15.65820145223120 & 2.65165678725683 & -0.27702271074802 \\
\hline $\mathrm{H}$ & 10.20849383987487 & 3.64416038570997 & 3.09640108821760 \\
\hline $\mathrm{H}$ & 8.27303508475799 & 4.87162833288370 & 0.71361699256309 \\
\hline $\mathrm{H}$ & 12.05890274726878 & 0.55017660118286 & -0.00409582941953 \\
\hline $\mathrm{H}$ & 12.44371011177862 & 3.65464150469056 & -1.06251106259286 \\
\hline $\mathrm{H}$ & 4.11125051900974 & 3.93622408910538 & 2.60749280332736 \\
\hline
\end{tabular}


Table IV: Continued. The atomic coordinates (in bohr) of the first excited state of 11-cis-retinal protonated Schiff base obtained at the B3LYP/TZVP level using the TDDFT method.

\begin{tabular}{|c|c|c|c|}
\hline Atom & $\mathrm{x}$ & $\mathrm{y}$ & z \\
\hline $\mathrm{H}$ & 3.69435451652292 & 1.11616894179002 & 4.35679560744975 \\
\hline $\mathrm{H}$ & 6.00345556229953 & 3.34356377431969 & 5.28447154555148 \\
\hline $\mathrm{H}$ & 9.16822065569346 & -0.16918125160718 & 5.32549322636191 \\
\hline $\mathrm{H}$ & 6.88440874766911 & -2.35906665237901 & 4.24570626694573 \\
\hline $\mathrm{H}$ & 9.80016088974609 & -2.04561883008953 & 2.63444266029858 \\
\hline $\mathrm{H}$ & 6.56835069177312 & -2.94640429420949 & -5.04572366688131 \\
\hline $\mathrm{H}$ & 3.93710854980090 & -0.96519220461009 & -4.86537269290079 \\
\hline $\mathrm{H}$ & 6.65631446409227 & -0.05051410916394 & -6.67773823438297 \\
\hline $\mathrm{H}$ & 4.35151008344861 & -3.75884662260046 & 0.39650964086244 \\
\hline $\mathrm{H}$ & 0.94463034832407 & 0.85072553037935 & -0.39772004179896 \\
\hline $\mathrm{H}$ & -1.98564217541313 & -6.65198991025233 & 0.96782353367505 \\
\hline $\mathrm{H}$ & 0.80821738292476 & -6.38341313702911 & -0.80718994115199 \\
\hline $\mathrm{H}$ & 0.84797337970491 & -5.79013958633067 & 2.46767611638173 \\
\hline $\mathrm{H}$ & -3.19664072560229 & 0.26600668041793 & -0.23866954053830 \\
\hline $\mathrm{H}$ & -5.38467090068103 & -5.05770569570270 & 0.69254179494597 \\
\hline $\mathrm{H}$ & -9.36818727829857 & -3.60329519629202 & 0.54672534218525 \\
\hline $\mathrm{H}$ & -8.47485828422658 & 4.32505602981314 & -0.64561570016336 \\
\hline $\mathrm{H}$ & -6.09994629399481 & 2.87344578468908 & 1.15849377824741 \\
\hline $\mathrm{H}$ & -12.68513336513070 & -1.27339369257430 & 0.25823812884505 \\
\hline $\mathrm{H}$ & -12.25261110397560 & 4.45816498181726 & -0.63218440358830 \\
\hline $\mathrm{H}$ & -16.62994231110980 & 4.26605742561368 & -0.50099739010077 \\
\hline $\mathrm{H}$ & -16.66275813174237 & 1.05946595229334 & -0.00404235130015 \\
\hline $\mathrm{H}$ & 10.71063637210005 & 0.59064097002053 & -4.45984304781381 \\
\hline $\mathrm{H}$ & 9.01612662963700 & 3.34854429821529 & -4.03624735458463 \\
\hline $\mathrm{H}$ & -6.21544748678690 & 2.41013139411953 & -2.14073362539823 \\
\hline
\end{tabular}


Table V: The atomic coordinates (in bohr) of the ground state of 11-cis-retinal protonated Schiff base obtained at the MP2/TZVP level.

\begin{tabular}{|c|c|c|c|}
\hline Atom & $\mathrm{x}$ & $\mathrm{y}$ & z \\
\hline $\mathrm{C}$ & 8.08345681287835 & -0.96406759982180 & 1.15089982105530 \\
\hline $\mathrm{C}$ & 9.90232521637256 & 1.04291651038877 & 2.16325940163250 \\
\hline $\mathrm{C}$ & 10.47936549456550 & 3.06309621220619 & 0.21289284163115 \\
\hline $\mathrm{C}$ & 8.05846744434652 & 4.46449913483776 & -0.43629608830853 \\
\hline $\mathrm{C}$ & 5.85380907083561 & 2.74208613889331 & -0.95298339344769 \\
\hline $\mathrm{C}$ & 5.85348792784954 & 0.28143934967987 & -0.19424504953803 \\
\hline $\mathrm{C}$ & 7.12234849649944 & -2.50307688550649 & 3.40873547795937 \\
\hline $\mathrm{C}$ & 9.47012787114245 & -2.73585920119979 & -0.67463191435054 \\
\hline $\mathrm{C}$ & 3.75050562587767 & 3.97132285186608 & -2.41625760476831 \\
\hline $\mathrm{C}$ & 3.75212491496593 & -1.41018268335215 & -0.67721800483380 \\
\hline $\mathrm{C}$ & 1.26351035108773 & -0.84933988199496 & -0.33860423480215 \\
\hline $\mathrm{C}$ & -0.76979895893857 & -2.56923689780707 & -0.79789923016062 \\
\hline $\mathrm{C}$ & -0.18952504337851 & -5.16055585249721 & -1.78732577384483 \\
\hline $\mathrm{C}$ & -3.18841637158202 & -1.72383567466173 & -0.27076522350678 \\
\hline $\mathrm{C}$ & -5.40297454204809 & -3.17293278498442 & -0.51638492129832 \\
\hline $\mathrm{C}$ & -7.89623145558580 & -2.43869964974945 & -0.21784883194075 \\
\hline $\mathrm{C}$ & -8.95000380921805 & 0.00503376423753 & -0.07016444402029 \\
\hline $\mathrm{C}$ & -7.40742684548306 & 2.32678781126671 & -0.59206303445147 \\
\hline $\mathrm{C}$ & -11.51055636564973 & 0.15506825727883 & 0.54974209750340 \\
\hline $\mathrm{C}$ & -12.86648764613868 & 2.37889132751684 & 0.78321924635730 \\
\hline $\mathrm{N}$ & -15.28845057201514 & 2.48091132615289 & 1.41688553673289 \\
\hline $\mathrm{H}$ & 11.62986379479142 & 0.10646845559043 & 2.80150064686465 \\
\hline $\mathrm{H}$ & 9.05162652360076 & 1.94422080146155 & 3.82152680219598 \\
\hline $\mathrm{H}$ & 11.26356569423783 & 2.20813833645963 & -1.49351566085418 \\
\hline $\mathrm{H}$ & 11.89220295277037 & 4.38352394241900 & 0.92803773573561 \\
\hline $\mathrm{H}$ & 5.95567586673956 & -1.33251405316638 & 4.64463619093706 \\
\hline
\end{tabular}


Table VI: Continued. The atomic coordinates (in bohr) of the ground state of 11-cis-retinal protonated Schiff base obtained at the MP2/TZVP level.

\begin{tabular}{|c|c|c|c|}
\hline Atom & $\mathrm{x}$ & $\mathrm{y}$ & $\mathrm{z}$ \\
\hline $\mathrm{H}$ & 6.00463442242949 & -4.13383496176459 & 2.81953969997166 \\
\hline $\mathrm{H}$ & 8.72455062785285 & -3.20458461889975 & 4.50427516541564 \\
\hline $\mathrm{H}$ & 11.01398578245933 & -3.68615378396821 & 0.31224887082138 \\
\hline $\mathrm{H}$ & 8.21974332493181 & -4.20034654467143 & -1.41412262959009 \\
\hline $\mathrm{H}$ & 10.25266721695225 & -1.70737622419129 & -2.27831146806245 \\
\hline $\mathrm{H}$ & 4.55069044855754 & 5.16207030636001 & -3.89675013726436 \\
\hline $\mathrm{H}$ & 2.47642963267478 & 2.61804012639349 & -3.29464228807887 \\
\hline $\mathrm{H}$ & 2.63402794642517 & 5.21339811679888 & -1.19600565419525 \\
\hline $\mathrm{H}$ & 4.24016345262802 & -3.32641947610846 & -1.22525447050206 \\
\hline $\mathrm{H}$ & 0.76836073876946 & 1.00142027561323 & 0.39160169028098 \\
\hline $\mathrm{H}$ & 0.76842243487986 & -5.02430860257807 & -3.60712142031957 \\
\hline $\mathrm{H}$ & 1.07472036537229 & -6.14415178503135 & -0.48981041488542 \\
\hline $\mathrm{H}$ & -1.86118897930177 & -6.31817390984937 & -2.05146520980246 \\
\hline $\mathrm{H}$ & -3.32836454611460 & 0.19218694267940 & 0.42188229491444 \\
\hline $\mathrm{H}$ & -5.15351291866966 & -5.17799780574710 & -0.85608455421677 \\
\hline $\mathrm{H}$ & -9.25096425378931 & -3.97594487688842 & -0.14722721218648 \\
\hline $\mathrm{H}$ & -8.57210286607304 & 3.92516857654406 & -1.14220562514733 \\
\hline $\mathrm{H}$ & -6.33161589383095 & 2.87395889374511 & 1.08118318486589 \\
\hline $\mathrm{H}$ & -12.51608160552150 & -1.60069710296243 & 0.88444910436071 \\
\hline $\mathrm{H}$ & -11.98419608622078 & 4.19798030546798 & 0.48044660053244 \\
\hline $\mathrm{H}$ & -16.195069973555163 & 4.14670599107227 & 1.57866178649606 \\
\hline $\mathrm{H}$ & -16.29783184004488 & 0.89694139940966 & 1.75052061928567 \\
\hline $\mathrm{H}$ & 8.34908163698718 & 5.68637449676452 & -2.07565459731719 \\
\hline $\mathrm{H}$ & 7.54041641683434 & 5.72563133272612 & 1.12750755483263 \\
\hline $\mathrm{H}$ & -6.07812728785827 & 1.97583966843407 & -2.12186260897289 \\
\hline
\end{tabular}


Table VII: The atomic coordinates (in bohr) of the ground state of 11-cis-blocked five-membered retinal protonated Schiff base. The structure was optimized at the B3LYP/TZVP level.

\begin{tabular}{|c|c|c|c|}
\hline Atom & $\mathrm{x}$ & $\mathrm{y}$ & z \\
\hline $\mathrm{C}$ & 4.75909004918404 & -0.45021168117948 & -2.64898976976816 \\
\hline $\mathrm{C}$ & 7.33376441715827 & -0.79985537916825 & -3.99411627822243 \\
\hline $\mathrm{C}$ & 9.34735733319705 & 1.03569760726358 & -3.08270703877271 \\
\hline $\mathrm{C}$ & 9.82858402016116 & 0.56242975897903 & -0.28864248234835 \\
\hline $\mathrm{C}$ & 7.48581268288573 & 0.19857424093669 & 1.27707545414454 \\
\hline $\mathrm{C}$ & 5.13001257947517 & -0.15177990847957 & 0.23656508638410 \\
\hline $\mathrm{C}$ & 3.21543563004895 & -2.85561514632356 & -3.25845223633845 \\
\hline $\mathrm{C}$ & 3.43873056267141 & 1.89955340062181 & -3.77855770123737 \\
\hline $\mathrm{C}$ & 8.04186412290092 & 0.33495221159029 & 4.07131261694121 \\
\hline $\mathrm{C}$ & 3.01838693462527 & -0.17713184234622 & 1.95869650846948 \\
\hline $\mathrm{C}$ & 0.46924580670285 & -0.13296191496206 & 1.51172269971799 \\
\hline $\mathrm{C}$ & -1.40152108180985 & -0.11646429513845 & 3.45323392851299 \\
\hline $\mathrm{C}$ & -0.60192977915534 & -0.18901888976779 & 6.18606817837388 \\
\hline $\mathrm{C}$ & -3.94840256273421 & -0.04183382093952 & 2.81134859254702 \\
\hline $\mathrm{C}$ & -6.03658427289355 & -0.01954067838996 & 4.45721480654622 \\
\hline $\mathrm{C}$ & -8.31188246808931 & 0.06242898949063 & 3.13971559993898 \\
\hline $\mathrm{C}$ & -7.85552016713806 & 0.09749541286105 & 0.53596788492033 \\
\hline $\mathrm{C}$ & -5.02338494620645 & 0.03366154586288 & 0.14489926231435 \\
\hline $\mathrm{C}$ & -9.72720614038267 & 0.17825390681812 & -1.33819942311776 \\
\hline $\mathrm{C}$ & -9.23500552566934 & 0.20834548696474 & -3.88783281608793 \\
\hline $\mathrm{N}$ & -10.98387693316842 & 0.28432288303153 & -5.70739751909996 \\
\hline $\mathrm{H}$ & 7.03143359831926 & -0.62026021746789 & -6.02944420968863 \\
\hline $\mathrm{H}$ & 8.00420927255297 & -2.73186808950927 & -3.67029539662840 \\
\hline $\mathrm{H}$ & 8.74538123469012 & 2.99017172314845 & -3.37799953015101 \\
\hline $\mathrm{H}$ & 11.09481382635136 & 0.79460657957863 & -4.15364910875600 \\
\hline $\mathrm{H}$ & 4.04234241350756 & -4.51021128030031 & -2.34309125050040 \\
\hline
\end{tabular}


Table VIII: Continued. The atomic coordinates (in bohr) of the ground state of 11-cis-blocked five-membered retinal protonated Schiff base. The structure was optimized at the B3LYP/TZVP level.

\begin{tabular}{|c|c|c|c|}
\hline Atom & $\mathrm{x}$ & $\mathrm{y}$ & $\mathrm{z}$ \\
\hline $\mathrm{H}$ & 1.24381545408030 & -2.74429968052013 & -2.68274148268584 \\
\hline $\mathrm{H}$ & 3.24518168249463 & -3.18560077470575 & -5.29660966549536 \\
\hline $\mathrm{H}$ & 3.12013349098054 & 1.61958011074250 & -5.80007295341459 \\
\hline $\mathrm{H}$ & 1.61609578646387 & 2.28991811884413 & -2.90308451541365 \\
\hline $\mathrm{H}$ & 4.59070276413610 & 3.59403390727814 & -3.54924710800053 \\
\hline $\mathrm{H}$ & 7.90142481088377 & 2.28757922792130 & 4.74515057002226 \\
\hline $\mathrm{H}$ & 6.82265220156631 & -0.83198411459132 & 5.24655968362107 \\
\hline $\mathrm{H}$ & 9.98492676558836 & -0.26363721344423 & 4.41642265302548 \\
\hline $\mathrm{H}$ & 3.54535213261328 & -0.15821408797955 & 3.93103312357673 \\
\hline $\mathrm{H}$ & -0.21665397893107 & -0.08478002274246 & -0.40614391618673 \\
\hline $\mathrm{H}$ & -2.19427788489815 & -0.16332922006764 & 7.47920633177667 \\
\hline $\mathrm{H}$ & 0.49272373234853 & -1.89400314590698 & 6.58145802520942 \\
\hline $\mathrm{H}$ & 0.59240680521213 & 1.43206582648699 & 6.64200191663113 \\
\hline $\mathrm{H}$ & -5.92634823386705 & -0.06084749306624 & 6.49554838717885 \\
\hline $\mathrm{H}$ & -10.16462724670745 & 0.09284551139261 & 3.99915066018391 \\
\hline $\mathrm{H}$ & -4.37845503222201 & 1.70294561112648 & -0.88983226402448 \\
\hline $\mathrm{H}$ & -11.68261948261950 & 0.21783094066416 & -0.71739364700769 \\
\hline $\mathrm{H}$ & -7.30269196238451 & 0.16963354988537 & -4.56973470974327 \\
\hline $\mathrm{H}$ & -10.49956168618938 & 0.30128796203140 & -7.54652096466784 \\
\hline $\mathrm{H}$ & -12.84604616992173 & 0.32501689504653 & -5.30015982719749 \\
\hline $\mathrm{H}$ & 10.93882097132998 & 2.09259561918437 & 0.55712216951457 \\
\hline $\mathrm{H}$ & 11.00150836619310 & -1.13605077622653 & -0.04407207728659 \\
\hline $\mathrm{H}$ & -4.46132558202393 & -1.62678613228630 & -0.95053435964054 \\
\hline
\end{tabular}


Table IX: The atomic coordinates (in bohr) of the first excited state of 11-cis-blocked five-membered retinal protonated Schiff base. The structure was optimized at the B3LYP/TZVP level using the TDDFT method.

\begin{tabular}{|c|c|c|c|}
\hline Atom & $\mathrm{x}$ & $\mathrm{y}$ & $\mathrm{z}$ \\
\hline $\mathrm{C}$ & 6.21702384419188 & -2.15532439864414 & -0.57080685603376 \\
\hline $\mathrm{C}$ & 8.77501425455660 & -1.74776651232691 & -1.91838187384857 \\
\hline $\mathrm{C}$ & 8.71188240255350 & 0.43571358545206 & -3.79618063454870 \\
\hline $\mathrm{C}$ & 8.02675380805887 & 2.87334907319508 & -2.42012919002312 \\
\hline $\mathrm{C}$ & 6.01516126495781 & 2.65750280964381 & -0.48427920657517 \\
\hline $\mathrm{C}$ & 5.06340687060269 & 0.27588740657768 & 0.32710346107780 \\
\hline $\mathrm{C}$ & 6.58184362885757 & -4.00469447654608 & 1.66272013390944 \\
\hline $\mathrm{C}$ & 4.26822717406852 & -3.36918877525541 & -2.43947826042326 \\
\hline $\mathrm{C}$ & 5.02912263862601 & 5.03508348108786 & 0.61862375554256 \\
\hline $\mathrm{C}$ & 2.93631139859217 & 0.19490296477570 & 2.16637453705418 \\
\hline $\mathrm{C}$ & 0.47552200918882 & 0.15208820187286 & 1.60635322176207 \\
\hline $\mathrm{C}$ & -1.59663637106436 & 0.05605117251562 & 3.44535062497511 \\
\hline $\mathrm{C}$ & -0.87279416052432 & 0.00373675305985 & 6.19538359065297 \\
\hline $\mathrm{C}$ & -4.04511306213015 & 0.01821800989584 & 2.66120121305241 \\
\hline $\mathrm{C}$ & -6.28255760660358 & -0.06385620071754 & 4.21048396147511 \\
\hline $\mathrm{C}$ & -8.42842880977499 & -0.08188852103138 & 2.73146394658432 \\
\hline $\mathrm{C}$ & -7.83348301620770 & -0.01775379284597 & 0.14384132498473 \\
\hline $\mathrm{C}$ & -4.97753156762222 & 0.04936927926259 & -0.08244906714060 \\
\hline $\mathrm{C}$ & -9.57840417863295 & -0.03280273877074 & -1.84603161850311 \\
\hline $\mathrm{C}$ & -8.98119409785855 & 0.01203929108424 & -4.36963944242614 \\
\hline $\mathrm{N}$ & -10.68924437023515 & 0.01038278413131 & -6.27809991905890 \\
\hline $\mathrm{H}$ & 9.31278096622144 & -3.50372102389720 & -2.85575658926858 \\
\hline $\mathrm{H}$ & 10.23103634365870 & -1.37954909839168 & -0.49348096668371 \\
\hline $\mathrm{H}$ & 7.34520523950622 & 0.07036321422020 & -5.30019091394816 \\
\hline $\mathrm{H}$ & 10.54177768189900 & 0.65537372690409 & -4.71830927839445 \\
\hline $\mathrm{H}$ & 7.83997923578303 & -3.21377264437723 & 3.09447019474702 \\
\hline
\end{tabular}


Table X: Continued. The atomic coordinates (in bohr) of the first excited state of 11-cis-blocked five-membered retinal protonated Schiff base. The structure was optimized at the B3LYP/TZVP level using the TDDFT method.

\begin{tabular}{|c|c|c|c|}
\hline Atom & $\mathrm{x}$ & $\mathrm{y}$ & $\mathrm{z}$ \\
\hline $\mathrm{H}$ & 4.79570234684177 & -4.49720482742476 & 2.55324052494439 \\
\hline $\mathrm{H}$ & 7.44863484178835 & -5.73391185609349 & 0.94902251362107 \\
\hline $\mathrm{H}$ & 5.07754713144311 & -5.14802065128183 & -3.09684803630396 \\
\hline $\mathrm{H}$ & 2.48783310582705 & -3.77107227945651 & -1.49587485820996 \\
\hline $\mathrm{H}$ & 3.90010691225022 & -2.18248640969165 & -4.08313143975547 \\
\hline $\mathrm{H}$ & 3.14228130742857 & 5.40483299875147 & -0.16043149475779 \\
\hline $\mathrm{H}$ & 4.75106651415893 & 4.89200670845762 & 2.65546291870599 \\
\hline $\mathrm{H}$ & 6.23221328153186 & 6.64151771593306 & 0.16753060187479 \\
\hline $\mathrm{H}$ & 3.56215079663528 & 0.15035336385897 & 4.12236821714402 \\
\hline $\mathrm{H}$ & -0.07209477514729 & 0.19610385744852 & -0.37058053274436 \\
\hline $\mathrm{H}$ & -2.50953531712114 & -0.03724569377039 & 7.43413839461062 \\
\hline $\mathrm{H}$ & 0.27459933859325 & -1.65989982218955 & 6.64046339629401 \\
\hline $\mathrm{H}$ & 0.24298475025769 & 1.66860448766828 & 6.71225506163237 \\
\hline $\mathrm{H}$ & -6.29468657709614 & -0.10786827845236 & 6.24782616128548 \\
\hline $\mathrm{H}$ & -10.33896646418745 & -0.14188397104801 & 3.45903826598887 \\
\hline $\mathrm{H}$ & -4.35174621282994 & 1.74691399651205 & -1.08472223170674 \\
\hline $\mathrm{H}$ & -11.56718348816908 & -0.08683075418269 & -1.33261411321636 \\
\hline $\mathrm{H}$ & -7.02794666328993 & 0.06268860129269 & -4.98007075683314 \\
\hline $\mathrm{H}$ & -10.14135887980408 & -0.01244691297734 & -8.09375813079426 \\
\hline $\mathrm{H}$ & -12.55768382553600 & -0.06879883997243 & -5.93112763437795 \\
\hline $\mathrm{H}$ & 7.55080559723790 & 4.42427436729775 & -3.70935886539595 \\
\hline $\mathrm{H}$ & 9.68837722112898 & 3.60797865405438 & -1.38860878767078 \\
\hline $\mathrm{H}$ & -4.27954553305217 & -1.58421237924347 & -1.14178528714350 \\
\hline
\end{tabular}


Table XI: The atomic coordinates (in bohr) of the ground state of all trans-polyene $\left(\mathrm{C}_{8} \mathrm{H}_{10}\right)$ obtained at the B3LYP/TZVP level.

\begin{tabular}{lccc}
\hline \hline Atom & $\mathrm{x}$ & $\mathrm{y}$ & $\mathrm{z}$ \\
\hline $\mathrm{H}$ & 9.86092763867384 & -0.67732845504617 & 0.00000000000000 \\
$\mathrm{C}$ & 8.11510564184498 & 0.38645336826194 & 0.00000000000000 \\
$\mathrm{H}$ & 8.27333697332723 & 2.42930034691367 & 0.00000000000000 \\
$\mathrm{C}$ & 5.86231389213062 & -0.76323013082965 & 0.00000000000000 \\
$\mathrm{C}$ & 3.46232327233650 & 0.53240196839169 & 0.00000000000000 \\
$\mathrm{C}$ & 1.19708501633632 & -0.63989963210065 & 0.00000000000000 \\
$\mathrm{C}$ & -1.19708501633632 & 0.63989963210064 & 0.00000000000000 \\
$\mathrm{H}$ & -1.15057416868926 & 2.69498067857261 & 0.00000000000000 \\
$\mathrm{C}$ & -3.46232327233650 & -0.53240196839169 & 0.00000000000000 \\
$\mathrm{C}$ & -5.86231389213062 & 0.76323013082965 & 0.00000000000000 \\
$\mathrm{C}$ & -8.11510564184498 & -0.38645336826193 & 0.00000000000000 \\
$\mathrm{H}$ & 5.79070357884699 & -2.81672110276780 & 0.00000000000000 \\
$\mathrm{H}$ & 3.51779711872947 & 2.58701339953816 & 0.00000000000000 \\
$\mathrm{H}$ & 1.15057416868926 & -2.69498067857261 & 0.00000000000000 \\
$\mathrm{H}$ & -3.51779711872947 & -2.58701339953816 & 0.00000000000000 \\
$\mathrm{H}$ & -5.79070357884699 & 2.81672110276780 & 0.00000000000000 \\
$\mathrm{H}$ & -9.86092763867384 & 0.67732845504617 & 0.00000000000000 \\
\hline & -8.27333697332723 & -2.42930034691367 & 0.00000000000000 \\
\hline
\end{tabular}


Table XII: The atomic coordinates (in bohr) of the first excited state of all trans-polyene $\left(\mathrm{C}_{8} \mathrm{H}_{10}\right)$ obtained at the B3LYP/TZVP level using the TDDFT method.

\begin{tabular}{lccc}
\hline \hline Atom & $\mathrm{x}$ & $\mathrm{y}$ & $\mathrm{z}$ \\
\hline $\mathrm{H}$ & 9.90493666802836 & -0.76064649984457 & 0.00000000000000 \\
$\mathrm{C}$ & 8.19520786260005 & 0.35988189958407 & 0.00000000000000 \\
$\mathrm{H}$ & 8.40805565965026 & 2.39781033868864 & 0.00000000000000 \\
$\mathrm{C}$ & 5.85886154840563 & -0.75079182600719 & 0.00000000000000 \\
$\mathrm{C}$ & 3.53234524513535 & 0.55247317261159 & 0.00000000000000 \\
$\mathrm{C}$ & 1.15713652498588 & -0.63451728281433 & 0.00000000000000 \\
$\mathrm{C}$ & -1.15713652498588 & 0.63451728281433 & 0.00000000000000 \\
$\mathrm{H}$ & -1.12369502155026 & 2.68968980606367 & 0.00000000000000 \\
$\mathrm{C}$ & -3.53234524513535 & -0.55247317261159 & 0.00000000000000 \\
$\mathrm{C}$ & -5.85886154840563 & 0.75079182600719 & 0.00000000000000 \\
$\mathrm{C}$ & -8.19520786260005 & -0.35988189958407 & 0.00000000000000 \\
$\mathrm{H}$ & 5.76550836713163 & -2.80477078702852 & 0.00000000000000 \\
$\mathrm{H}$ & 3.57997160232034 & 2.60705923283450 & 0.00000000000000 \\
$\mathrm{H}$ & 1.12369502155026 & -2.68968980606367 & 0.00000000000000 \\
$\mathrm{H}$ & -3.57997160232035 & -2.60705923283450 & 0.00000000000000 \\
$\mathrm{H}$ & -5.76550836713163 & 2.80477078702852 & 0.00000000000000 \\
$\mathrm{H}$ & -9.90493666802836 & 0.76064649984457 & 0.00000000000000 \\
\hline & -8.40805565965026 & -2.39781033868864 & 0.00000000000000 \\
\hline
\end{tabular}


Table XIII: The atomic coordinates (in bohr) of the ground state of all trans-polyene $\left(\mathrm{C}_{8} \mathrm{H}_{10}\right)$ obtained at the MP2/TZVP level.

\begin{tabular}{lccc}
\hline \hline Atom & $\mathrm{x}$ & $\mathrm{y}$ & $\mathrm{z}$ \\
\hline $\mathrm{H}$ & 9.85574880636888 & -0.65923737404704 & 0.00000000000000 \\
$\mathrm{C}$ & 8.10335826702398 & 0.39510838066040 & 0.00000000000000 \\
$\mathrm{H}$ & 8.22618651351313 & 2.44049238658800 & 0.00000000000000 \\
$\mathrm{C}$ & 5.85175942889262 & -0.77746505885545 & 0.00000000000000 \\
$\mathrm{C}$ & 3.45947671064126 & 0.53639165327818 & 0.00000000000000 \\
$\mathrm{C}$ & 1.19320683027847 & -0.64953670109784 & 0.00000000000000 \\
$\mathrm{C}$ & -1.19320683027847 & 0.64953670109784 & 0.00000000000000 \\
$\mathrm{H}$ & -1.15081807032542 & 2.70660564483477 & 0.00000000000000 \\
$\mathrm{C}$ & -3.45947671064126 & -0.53639165327818 & 0.00000000000000 \\
$\mathrm{C}$ & -5.85175942889262 & 0.77746505885545 & 0.00000000000000 \\
$\mathrm{C}$ & -8.10335826702398 & -0.39510838066040 & 0.00000000000000 \\
$\mathrm{H}$ & 5.78456485605974 & -2.83122266980335 & 0.00000000000000 \\
$\mathrm{H}$ & 3.50851160541631 & 2.59309545440901 & 0.00000000000000 \\
$\mathrm{H}$ & 1.15081807032542 & -2.70660564483477 & 0.00000000000000 \\
$\mathrm{H}$ & -3.50851160541631 & -2.59309545440901 & 0.00000000000000 \\
$\mathrm{H}$ & -5.78456485605974 & 2.83122266980335 & 0.00000000000000 \\
$\mathrm{H}$ & -9.85574880636888 & 0.65923737404705 & 0.00000000000000 \\
\hline & -8.22618651351313 & -2.44049238658800 & 0.00000000000000 \\
\hline
\end{tabular}


Table XIV: The atomic coordinates (in bohr) of the first excited state of all trans-polyene $\left(\mathrm{C}_{8} \mathrm{H}_{10}\right)$ obtained at the CC2/TZVP level.

\begin{tabular}{lccc}
\hline \hline Atom & $\mathrm{x}$ & $\mathrm{y}$ & $\mathrm{z}$ \\
\hline $\mathrm{H}$ & 9.90010199735357 & -0.76679390232837 & 0.00000000000000 \\
$\mathrm{C}$ & 8.19044859002536 & 0.35959300610032 & 0.00000000000000 \\
$\mathrm{H}$ & 8.38215556887951 & 2.40281999167925 & 0.00000000000000 \\
$\mathrm{C}$ & 5.83821856132613 & -0.75877709847117 & 0.00000000000000 \\
$\mathrm{C}$ & 3.53417169978318 & 0.56162905111322 & 0.00000000000000 \\
$\mathrm{C}$ & 1.14754954831740 & -0.63833747681305 & 0.00000000000000 \\
$\mathrm{C}$ & -1.14754954831740 & 0.63833747681305 & 0.00000000000000 \\
$\mathrm{H}$ & -1.11739167105223 & 2.69867544330914 & 0.00000000000000 \\
$\mathrm{C}$ & -3.53417169978318 & -0.56162905111322 & 0.00000000000000 \\
$\mathrm{C}$ & -5.83821856132613 & 0.75877709847117 & 0.00000000000000 \\
$\mathrm{C}$ & -8.19044859002536 & -0.35959300610032 & 0.00000000000000 \\
$\mathrm{H}$ & 5.73605996599149 & -2.81523646377100 & 0.00000000000000 \\
$\mathrm{H}$ & 3.57887227719371 & 2.62166485510343 & 0.00000000000000 \\
$\mathrm{H}$ & 1.11739167105223 & -2.69867544330914 & 0.00000000000000 \\
$\mathrm{H}$ & -3.57887227719371 & -2.62166485510343 & 0.00000000000000 \\
$\mathrm{H}$ & -5.73605996599149 & 2.81523646377100 & 0.00000000000000 \\
$\mathrm{H}$ & -9.90010199735357 & 0.76679390232837 & 0.00000000000000 \\
\hline & -8.38215556887951 & -2.40281999167925 & 0.00000000000000 \\
\hline
\end{tabular}


Table XV: The atomic coordinates (in bohr) of the ground state of 2-cis $\mathrm{C}_{7} \mathrm{H}_{8} \mathrm{NH}_{2}^{+}$obtained at the B3LYP/TZVP level.

\begin{tabular}{|c|c|c|c|}
\hline Atom & $\mathrm{x}$ & $\mathrm{y}$ & $\mathrm{z}$ \\
\hline $\mathrm{H}$ & 8.38431261793928 & 3.18914933091960 & -0.00187833236634 \\
\hline $\mathrm{C}$ & 7.50252051207073 & 1.34447395464289 & 0.00392820197167 \\
\hline $\mathrm{H}$ & 8.74942918610226 & -0.27992780893837 & 0.01602071920395 \\
\hline $\mathrm{C}$ & 4.97800482324822 & 1.07578352064198 & -0.00341606393687 \\
\hline $\mathrm{C}$ & 3.82586003835697 & -1.37137591482817 & 0.00288653964905 \\
\hline $\mathrm{C}$ & 1.30220161250754 & -1.95880088790677 & -0.00201676901657 \\
\hline $\mathrm{C}$ & -0.68385440608698 & -0.18686902659470 & -0.00446394330883 \\
\hline $\mathrm{H}$ & -0.21263864427092 & 1.80963878530483 & -0.00579323037319 \\
\hline $\mathrm{C}$ & -3.21006386257353 & -0.82218546545867 & -0.00266528347037 \\
\hline $\mathrm{C}$ & -5.06468914170320 & 1.04604865367300 & -0.00006024019167 \\
\hline $\mathrm{N}$ & -7.51568578974726 & 0.62513541855425 & 0.00403789394762 \\
\hline $\mathrm{H}$ & 3.80005486053975 & 2.75076013821133 & -0.01565255232609 \\
\hline $\mathrm{H}$ & 5.13169723952068 & -2.95464250432335 & 0.01037224634347 \\
\hline $\mathrm{H}$ & 0.79018323973000 & -3.94249018024497 & 0.00074303910898 \\
\hline $\mathrm{H}$ & -3.77191417501154 & -2.79406765309504 & -0.00135102337072 \\
\hline $\mathrm{H}$ & -4.50922336998727 & 3.01991962660065 & -0.00088277744143 \\
\hline $\mathrm{H}$ & -8.76767960642029 & 2.06253295342197 & 0.00631218419583 \\
\hline $\mathrm{H}$ & -8.23132962602175 & -1.14579105000978 & 0.00520325635863 \\
\hline
\end{tabular}


Table XVI: The atomic coordinates (in bohr) of the first excited state of 2 - cis $\mathrm{C}_{7} \mathrm{H}_{8} \mathrm{NH}_{2}^{+}$obtained at the B3LYP/TZVP level using the TDDFT method.

\begin{tabular}{|c|c|c|c|}
\hline Atom & $\mathrm{x}$ & $\mathrm{y}$ & $\mathrm{z}$ \\
\hline $\mathrm{H}$ & 6.97049639928585 & 4.21303824522234 & -0.68111532127422 \\
\hline $\mathrm{C}$ & 6.63424122318926 & 2.31159803302657 & -0.01192264609494 \\
\hline $\mathrm{H}$ & 8.19050143378342 & 1.33426993573364 & 0.88886341799431 \\
\hline $\mathrm{C}$ & 4.30815402925148 & 1.17166476175165 & -0.29832877959643 \\
\hline $\mathrm{C}$ & 3.84452418508317 & -1.30949556865370 & 0.55039939260752 \\
\hline $\mathrm{C}$ & 1.56201084498957 & -2.54534913520293 & 0.28182606763338 \\
\hline $\mathrm{C}$ & -0.75704385617160 & -1.53581929878625 & -0.91992803847664 \\
\hline $\mathrm{H}$ & -0.94844896944582 & -1.99704098901726 & -2.90818782407630 \\
\hline $\mathrm{C}$ & -2.68497943937316 & -0.41410562885834 & 0.36140940731600 \\
\hline $\mathrm{C}$ & -4.98334509266826 & 0.36661272603458 & -0.71771118549228 \\
\hline $\mathrm{N}$ & -6.85408269825059 & 1.49031553770164 & 0.61206904261075 \\
\hline $\mathrm{H}$ & 2.79988193867095 & 2.20490730023289 & -1.20987289116931 \\
\hline $\mathrm{H}$ & 5.38679076383214 & -2.31571644436172 & 1.45311886362827 \\
\hline $\mathrm{H}$ & 1.50946190182225 & -4.47483402286484 & 0.98417727413840 \\
\hline $\mathrm{H}$ & -2.47222814164354 & -0.08845372527403 & 2.37882555287239 \\
\hline $\mathrm{H}$ & -5.34330535842215 & 0.10234152380560 & -2.70970378502387 \\
\hline $\mathrm{H}$ & -8.51145758829996 & 1.90891424080572 & -0.21180481596310 \\
\hline $\mathrm{H}$ & -6.75628122363643 & 1.69809111943321 & 2.49826535729600 \\
\hline
\end{tabular}


Table XVII: The atomic coordinates (in bohr) of the ground state of 2-cis $\mathrm{C}_{7} \mathrm{H}_{8} \mathrm{NH}_{2}^{+}$obtained at the MP2/TZVP level.

\begin{tabular}{|c|c|c|c|}
\hline Atom & $\mathrm{x}$ & $\mathrm{y}$ & $\mathrm{z}$ \\
\hline $\mathrm{H}$ & 8.27884218061977 & 3.24910873211499 & -0.00077768911618 \\
\hline $\mathrm{C}$ & 7.42297106207638 & 1.39272490086717 & 0.00636878471814 \\
\hline $\mathrm{H}$ & 8.67330733858930 & -0.22865906348508 & 0.02306702452853 \\
\hline $\mathrm{C}$ & 4.89874239678938 & 1.09317692215610 & -0.00477366561772 \\
\hline $\mathrm{C}$ & 3.81289534782505 & -1.38661704990112 & 0.00383470943463 \\
\hline $\mathrm{C}$ & 1.30368439284820 & -2.00415797589336 & -0.00337684678408 \\
\hline $\mathrm{C}$ & -0.67387874775827 & -0.21618092968868 & -0.00723608969898 \\
\hline $\mathrm{H}$ & -0.20175037093078 & 1.78200380395200 & -0.01104244196642 \\
\hline $\mathrm{C}$ & -3.18448211365065 & -0.85859110698338 & -0.00310505301189 \\
\hline $\mathrm{C}$ & -5.00771932152566 & 1.05111911521761 & -0.00150133488680 \\
\hline $\mathrm{N}$ & -7.44884535264699 & 0.66451865556346 & 0.00690458226993 \\
\hline $\mathrm{H}$ & 3.69663846356856 & 2.74901492951084 & -0.02150212985300 \\
\hline $\mathrm{H}$ & 5.15460255030038 & -2.94196465898008 & 0.01426879556022 \\
\hline $\mathrm{H}$ & 0.79205516442115 & -3.98810059008806 & 0.00149174980815 \\
\hline $\mathrm{H}$ & -3.76230862444147 & -2.82513538477477 & 0.00125414963652 \\
\hline $\mathrm{H}$ & -4.41046304655170 & 3.01363595174675 & -0.00680660817448 \\
\hline $\mathrm{H}$ & -8.68069073958744 & 2.12389151123541 & 0.00784420279289 \\
\hline $\mathrm{H}$ & -8.17947235115714 & -1.10343013692135 & 0.01291059736757 \\
\hline
\end{tabular}


Table XVIII: The atomic coordinates (in bohr) of the first excited state of 2-cis $\mathrm{C}_{7} \mathrm{H}_{8} \mathrm{NH}_{2}^{+}$obtained at the CC2/TZVP level.

\begin{tabular}{|c|c|c|c|}
\hline Atom & $\mathrm{x}$ & $\mathrm{y}$ & $\mathrm{z}$ \\
\hline $\mathrm{H}$ & 6.18484613443144 & 4.51268472176881 & -0.99968084270745 \\
\hline $\mathrm{C}$ & 6.04253999668778 & 2.66656758154959 & -0.12032045968969 \\
\hline $\mathrm{H}$ & 7.62192145338062 & 2.01514822932961 & 1.01651327562672 \\
\hline $\mathrm{C}$ & 3.91805507805867 & 1.17809492449477 & -0.42709057393537 \\
\hline $\mathrm{C}$ & 3.79781410412933 & -1.23969241145971 & 0.64060855761737 \\
\hline $\mathrm{C}$ & 1.63025555635669 & -2.75315310533180 & 0.37448207408149 \\
\hline $\mathrm{C}$ & -0.67494985010269 & -1.94190811659956 & -0.89069534755083 \\
\hline $\mathrm{H}$ & -1.16581027112389 & -2.90665934714934 & -2.64393182093728 \\
\hline $\mathrm{C}$ & -2.42150494452377 & -0.40317290253778 & 0.34723025379900 \\
\hline $\mathrm{C}$ & -4.79606185072129 & 0.17775214356900 & -0.60819256609778 \\
\hline $\mathrm{N}$ & -6.45688442660080 & 1.76777468293862 & 0.53500090545642 \\
\hline $\mathrm{H}$ & 2.31747562744106 & 1.84918564745171 & -1.52343364150354 \\
\hline $\mathrm{H}$ & 5.39735428460851 & -1.95555298379505 & 1.71499902312634 \\
\hline $\mathrm{H}$ & 1.72269493752172 & -4.62880744828022 & 1.23506173468765 \\
\hline $\mathrm{H}$ & -1.89763676211193 & 0.39420111465923 & 2.17512992170254 \\
\hline $\mathrm{H}$ & -5.39619433349735 & -0.57719234655606 & -2.42035079607378 \\
\hline $\mathrm{H}$ & -8.22028871339156 & 1.95738579908764 & -0.16126042377368 \\
\hline $\mathrm{H}$ & -6.16525924084582 & 2.36675121282877 & 2.32301249446544 \\
\hline
\end{tabular}


Table XIX: The atomic coordinates (in bohr) of the ground state of the $\beta$-ionone ring with the retinyl chain replaced by a $-\mathrm{CH}=\mathrm{NH}_{2}^{+}$unit. The structure was optimized at the B3LYP/TZVP level.

\begin{tabular}{|c|c|c|c|}
\hline Atom & $\mathrm{x}$ & $\mathrm{y}$ & $\mathrm{z}$ \\
\hline $\mathrm{C}$ & 1.90903491891294 & -1.51071527340314 & 0.09929736849575 \\
\hline $\mathrm{C}$ & 3.79741401691067 & 0.58672190723453 & 0.83524845781382 \\
\hline $\mathrm{C}$ & 3.33834827671240 & 3.05632503058790 & -0.56596492038627 \\
\hline $\mathrm{C}$ & 0.71382094412513 & 4.04889746963202 & 0.07112225498805 \\
\hline $\mathrm{C}$ & -1.34256589654618 & 2.11131487380811 & 0.12691292822982 \\
\hline $\mathrm{C}$ & -0.82386954792833 & -0.43969369376875 & 0.06051756560845 \\
\hline $\mathrm{C}$ & 2.15294996135268 & -3.63029796311872 & 2.09309013940268 \\
\hline $\mathrm{C}$ & 2.57058326579746 & -2.58544206498965 & -2.53059527735480 \\
\hline $\mathrm{C}$ & -3.95236165450308 & 3.22115886127347 & 0.29041874570680 \\
\hline $\mathrm{C}$ & -2.72996571538457 & -2.32349144760537 & -0.13008105464694 \\
\hline $\mathrm{N}$ & -5.19918384443581 & -2.14348394397719 & -0.27824116544232 \\
\hline $\mathrm{H}$ & -6.25935386904198 & -3.72498368972761 & -0.44379709899124 \\
\hline $\mathrm{H}$ & 5.70828529648761 & -0.10774521333919 & 0.48558637585593 \\
\hline $\mathrm{H}$ & 3.66446229950922 & 0.93169484707971 & 2.87201617262037 \\
\hline $\mathrm{H}$ & 3.51098577847296 & 2.78355698097288 & -2.60575598980319 \\
\hline $\mathrm{H}$ & 4.75407658771009 & 4.46194977505802 & -0.04570454310325 \\
\hline $\mathrm{H}$ & 1.48504722689057 & -3.01216911515469 & 3.94585712092457 \\
\hline $\mathrm{H}$ & 1.15107891642637 & -5.36180372183532 & 1.58174627673327 \\
\hline $\mathrm{H}$ & 4.13875050592979 & -4.15203664539962 & 2.28592864198593 \\
\hline $\mathrm{H}$ & 4.46520411751284 & -3.40102305753818 & -2.50563092324733 \\
\hline $\mathrm{H}$ & 1.27034787936626 & -4.08759528357614 & -3.10107333760545 \\
\hline $\mathrm{H}$ & 2.51597345491862 & -1.13586166210581 & -3.99621792096009 \\
\hline $\mathrm{H}$ & -3.85990042551950 & 5.24885092881808 & 0.62510203564728 \\
\hline $\mathrm{H}$ & -4.98243614893193 & 2.97323920705607 & -1.49272462320466 \\
\hline $\mathrm{H}$ & -5.06248221954280 & 2.40272760781602 & 1.83122923226573 \\
\hline $\mathrm{H}$ & -2.07589375335296 & -4.26229337598908 & -0.20873650190479 \\
\hline $\mathrm{H}$ & -6.14033639891828 & -0.48667329271120 & -0.25424267520486 \\
\hline $\mathrm{H}$ & 0.1260688902352120 & 5.55686153840150 & -1.22233622784663 \\
\hline $\mathrm{H}$ & 0.70925560384449 & 4.95418593547043 & 1.94491946149236 \\
\hline
\end{tabular}


Table XX: The atomic coordinates (in bohr) of the first excited state of the $\beta$-ionone ring with the retinyl chain replaced by a $-\mathrm{CH}=\mathrm{NH}_{2}^{+}$unit. The structure was optimized at the B3LYP/TZVP level using the TDDFT method.

\begin{tabular}{|c|c|c|c|}
\hline Atom & $\mathrm{x}$ & $\mathrm{y}$ & $\mathrm{z}$ \\
\hline $\mathrm{C}$ & 1.03995657091522 & -1.99334567337589 & 0.03328538935449 \\
\hline $\mathrm{C}$ & 3.53554871405662 & -1.01384827356754 & 1.19130300196527 \\
\hline $\mathrm{C}$ & 4.37910119160934 & 1.49914433991809 & 0.07147603626816 \\
\hline $\mathrm{C}$ & 2.31719087426182 & 3.48297413561365 & 0.45425222913387 \\
\hline $\mathrm{C}$ & -0.29584923130460 & 2.61880300190243 & -0.09254302594725 \\
\hline $\mathrm{C}$ & -0.90079920477578 & 0.00981885365325 & -0.27267791816232 \\
\hline $\mathrm{C}$ & 0.07093346202281 & -4.27746379085044 & 1.57729750815339 \\
\hline $\mathrm{C}$ & 1.54771314804655 & -2.97337281564777 & -2.75961982315611 \\
\hline $\mathrm{C}$ & -2.26281527535295 & 4.59744959119152 & -0.32300062527247 \\
\hline $\mathrm{C}$ & -3.54281660822665 & -0.81494766049773 & -0.94309738479897 \\
\hline $\mathrm{N}$ & -5.36882166366481 & -0.79427316414504 & 0.90454235903569 \\
\hline $\mathrm{H}$ & -7.10498195876727 & -1.39362081777041 & 0.39199615613199 \\
\hline $\mathrm{H}$ & 4.98961081117968 & -2.45428036728355 & 0.94679318911395 \\
\hline $\mathrm{H}$ & 3.27005827915852 & -0.79667871940324 & 3.23316186767843 \\
\hline $\mathrm{H}$ & 4.79689821945078 & 1.30878419111722 & -1.94139078421087 \\
\hline $\mathrm{H}$ & 6.12145724338415 & 2.14136306086792 & 0.96517921542467 \\
\hline $\mathrm{H}$ & -0.35204942220653 & -3.72912131408112 & 3.52196387674953 \\
\hline $\mathrm{H}$ & -1.59256334632389 & -5.14935315383257 & 0.74202376068731 \\
\hline $\mathrm{H}$ & 1.56102322962157 & -5.70100978145037 & 1.64969030877770 \\
\hline $\mathrm{H}$ & 2.96686760662597 & -4.46198330291452 & -2.59519682253227 \\
\hline $\mathrm{H}$ & -0.15131657243865 & -3.76865877691600 & -3.59640718517692 \\
\hline $\mathrm{H}$ & 2.28012165196399 & -1.49461573824742 & -3.98961444854067 \\
\hline $\mathrm{H}$ & -1.57953772020133 & 6.17916328170024 & -1.46369784257833 \\
\hline $\mathrm{H}$ & -4.05021977695668 & 3.91717350750680 & -1.05737890160183 \\
\hline $\mathrm{H}$ & -2.62200211825902 & 5.40060099252956 & 1.56147311992815 \\
\hline $\mathrm{H}$ & -4.17342272771338 & -0.50334834226142 & -2.85676819138148 \\
\hline $\mathrm{H}$ & -4.90668004491546 & -1.29623496239974 & 2.68429332471523 \\
\hline $\mathrm{H}$ & 2.6983152176704021 & 5.24486716880836 & -0.55744225075072 \\
\hline $\mathrm{H}$ & 2.28888858233847 & 4.06674782745294 & 2.46261227957698 \\
\hline
\end{tabular}


Table XXI: The atomic coordinates (in bohr) of the ground state of the $\beta$-ionone ring with the retinyl chain replaced by a $-\mathrm{CH}=\mathrm{NH}_{2}^{+}$unit. The structure was optimized at the MP2/TZVP level.

\begin{tabular}{|c|c|c|c|}
\hline Atom & $\mathrm{x}$ & $\mathrm{y}$ & $\mathrm{z}$ \\
\hline $\mathrm{C}$ & 1.94612707634829 & -1.44117461117334 & 0.12832512289754 \\
\hline $\mathrm{C}$ & 3.77771913259968 & 0.72912550137396 & 0.68500167868640 \\
\hline $\mathrm{C}$ & 3.14844440990766 & 3.10223496353078 & -0.79558684066492 \\
\hline $\mathrm{C}$ & 0.58263002477223 & 4.05337672762783 & 0.05180749639945 \\
\hline $\mathrm{C}$ & -1.40007772632125 & 2.05324051910413 & 0.19070936631488 \\
\hline $\mathrm{C}$ & -0.77941931726469 & -0.46503997567858 & 0.11468702344491 \\
\hline $\mathrm{C}$ & 2.30573145887995 & -3.43156477874915 & 2.20592363643384 \\
\hline $\mathrm{C}$ & 2.53176268883834 & -2.63050284267730 & -2.44535611749540 \\
\hline $\mathrm{C}$ & -4.02768959622633 & 3.06804742252698 & 0.49492487037558 \\
\hline $\mathrm{C}$ & -2.64011833735324 & -2.41127978115655 & 0.03275721938254 \\
\hline $\mathrm{N}$ & -5.02738301656134 & -2.23583761191321 & -0.55456721019619 \\
\hline $\mathrm{H}$ & -6.12974216448924 & -3.80052476046778 & -0.56737490071550 \\
\hline $\mathrm{H}$ & 5.69462359318750 & 0.07587259068982 & 0.27780876438765 \\
\hline $\mathrm{H}$ & 3.71135721350015 & 1.16406947481784 & 2.71035183505600 \\
\hline $\mathrm{H}$ & 3.12452180143492 & 2.71630516642367 & -2.82476702057556 \\
\hline $\mathrm{H}$ & 4.57290717390876 & 4.56044331908117 & -0.48472886813122 \\
\hline $\mathrm{H}$ & 1.62599853510623 & -2.74826842174521 & 4.03241888034742 \\
\hline $\mathrm{H}$ & 1.39437533873836 & -5.23567407518928 & 1.78604974823118 \\
\hline $\mathrm{H}$ & 4.32150341233716 & -3.83270310476317 & 2.39299059699312 \\
\hline $\mathrm{H}$ & 4.44706650799622 & -3.40027924157005 & -2.44796996404385 \\
\hline $\mathrm{H}$ & 1.23875440328890 & -4.18853541167401 & -2.86886794639408 \\
\hline $\mathrm{H}$ & 2.38012605592544 & -1.26005679173506 & -3.97956957664346 \\
\hline $\mathrm{H}$ & -3.94343897577694 & 4.96605215289782 & 1.29208823463404 \\
\hline $\mathrm{H}$ & -4.95437911299207 & 3.27356650610092 & -1.35035352740917 \\
\hline $\mathrm{H}$ & -5.21648011252180 & 1.92059152313676 & 1.73106986347995 \\
\hline $\mathrm{H}$ & -2.02848858900589 & -4.32832199150143 & 0.42945820070576 \\
\hline $\mathrm{H}$ & -5.83249272093217 & -0.58741079712982 & -1.08878001865514 \\
\hline $\mathrm{H}$ & -0.13807759722930 & 5.55991533803481 & -1.17573501723789 \\
\hline $\mathrm{H}$ & $0.70722656124775^{22}$ & 4.91664208459556 & 1.93941723573344 \\
\hline
\end{tabular}


Table XXII: The atomic coordinates (in bohr) of the first excited state of the $\beta$-ionone ring with the retinyl chain replaced by a $-\mathrm{CH}=\mathrm{NH}_{2}^{+}$unit. The structure was optimized at the $\mathrm{CC} 2 / \mathrm{TZVP}$ level.

\begin{tabular}{|c|c|c|c|}
\hline Atom & $\mathrm{x}$ & $\mathrm{y}$ & $\mathrm{z}$ \\
\hline $\mathrm{C}$ & 1.78509497369578 & -1.49872904004944 & 0.30909292537650 \\
\hline $\mathrm{C}$ & 3.85819238361295 & 0.50995538368704 & 0.33986049320193 \\
\hline $\mathrm{C}$ & 3.21545541757098 & 2.73066603583168 & -1.36911406877833 \\
\hline $\mathrm{C}$ & 0.86311037162904 & 4.01618282327526 & -0.34633777543844 \\
\hline $\mathrm{C}$ & -1.17449469064204 & 2.27546049727043 & 0.43514966801113 \\
\hline $\mathrm{C}$ & -0.78985841424541 & -0.39440646374881 & 0.43027721516527 \\
\hline $\mathrm{C}$ & 2.09947840194113 & -3.32470318054835 & 2.56109693379237 \\
\hline $\mathrm{C}$ & 1.87862688463286 & -3.09704359173141 & -2.14976446786613 \\
\hline $\mathrm{C}$ & -3.66099644637114 & 3.31792166070315 & 1.14280638420371 \\
\hline $\mathrm{C}$ & -3.00277723071265 & -2.09837903181630 & 0.50645848142516 \\
\hline $\mathrm{N}$ & -4.66176926765305 & -1.99515040512804 & -1.56472311542480 \\
\hline $\mathrm{H}$ & -6.11928627810906 & -3.23807165068312 & -1.43389620452162 \\
\hline $\mathrm{H}$ & 5.63991329481867 & -0.37536546178432 & -0.21833470300665 \\
\hline $\mathrm{H}$ & 4.11570324948124 & 1.19694134103330 & 2.27995602270025 \\
\hline $\mathrm{H}$ & 2.88902945151657 & 2.09739999154933 & -3.30987218087653 \\
\hline $\mathrm{H}$ & 4.76962539595515 & 4.08491576367592 & -1.43897018899245 \\
\hline $\mathrm{H}$ & 1.92085907076046 & -2.32286916041813 & 4.35864897401136 \\
\hline $\mathrm{H}$ & 0.70881283088487 & -4.84313064300883 & 2.48543961743039 \\
\hline $\mathrm{H}$ & 3.99383042971895 & -4.14079492063892 & 2.45972363924430 \\
\hline $\mathrm{H}$ & 3.73676689374607 & -3.99554466808893 & -2.23159995527442 \\
\hline $\mathrm{H}$ & 0.43461834900416 & -4.56705723589464 & -2.11535942236743 \\
\hline $\mathrm{H}$ & 1.63894269452807 & -1.94019759574992 & -3.84269485814870 \\
\hline $\mathrm{H}$ & -3.57564745469966 & 5.34543552116446 & 1.48633972977631 \\
\hline $\mathrm{H}$ & -4.94690911499704 & 2.95381666750936 & -0.45504136687628 \\
\hline $\mathrm{H}$ & -4.49276245482061 & 2.32163043507066 & 2.74879016487662 \\
\hline $\mathrm{H}$ & -3.90846534290966 & -2.31648077905683 & 2.34454455747559 \\
\hline $\mathrm{H}$ & -3.82199216274133 & -2.11036231679387 & -3.28912554124055 \\
\hline $\mathrm{H}$ & 0.0509001176592423 & 5.43308701431455 & -1.62932476236304 \\
\hline $\mathrm{H}$ & 1.30892129885076 & 5.10232321417656 & 1.38564912233298 \\
\hline
\end{tabular}

